\title{
A Novel Adjustable Plasmonic Filter Realization by Split Mode Ring Resonators
}

\author{
Mohsen Janipour, Mohammad Azim Karami, Rahman Sofiani, Farrokh Hodjat Kashani \\ Department of Electrical Engineering, Iran University of Science and Technology, Tehran, Iran. \\ Email: karami@iust.ac.ir
}

Received September $4^{\text {th }}, 2013$; revised October $7^{\text {th }}, 2013$; accepted November $5^{\text {th }}, 2013$

Copyright (C) 2013 Mohsen Janipour et al. This is an open access article distributed under the Creative Commons Attribution License, which permits unrestricted use, distribution, and reproduction in any medium, provided the original work is properly cited. In accordance of the Creative Commons Attribution License all Copyrights (C) 2013 are reserved for SCIRP and the owner of the intellectual property Mohsen Janipour et al. All Copyright (C) 2013 are guarded by law and by SCIRP as a guardian.

\begin{abstract}
A novel nanoscale plasmonic filter consisting of two coupled metal-insulator-metal (MIM) waveguides is introduced. The new structure functionality is verified by numerical simulations in different configurations of the filter. The impedance variation characteristic named as split mode ring resonancy is achieved by partially narrowing or expanding the waveguide diameter. The main parameters of the filter are evaluated by using the parameters of an implemented type of ring resonator. Moreover, modal analysis for Surface Plasmon Polariton (SPP) propagation is performed while changing the main spatial parameters of the device.
\end{abstract}

Keywords: Plasmonic Filter; Ring Resonator; MIM Waveguide

\section{Introduction}

Plasmonics and integrated optical devices play an important role in routing, switching and controlling of light in the various fields of modern optical communication and networks [1]. Plasmonic waveguides transfer the electromagnetic waves coupled to surface collective oscillations of free electrons on a metal, called Surface Plasmon Polaritons (SPPs). The SPP waves are bound to and propagate along metal-dielectric-metal interfaces [2]. The light confinement below the diffraction limit is the main identity of plasmonic waveguide making them different from other types of optical waveguides such as photonic crystals, optical fibers, etc. Furthermore, the introduction of deep-submicron technologies in electronic engineering leads to a revolution in optoelectronic circuits [3]. Various structures such as nano-particle plasmon waveguides [4-6], Domino plasmon waveguide [7], grooves and wedges [8-10] and Metal-Insulator-Metal waveguides (MIM) [11,12], are introduced by transporting SPPs. In addition to the fabrication simplicity, high degree of SPP confinement and compact size have made MIM geometry the most attractive among other nanosclae waveguides [13]. Different types of plasmonic filters such as tooth shaped subwavelength, and add-drop topologies are introduced and analyzed by numerical methods such as Finite-Difference Time-Domain (FDTD) $[14,15]$. It has been shown that the transmission spectra of an asymmetrical multiple teeth-shaped structure can present a narrow band pass filter [16]. Moreover, ring resonator filters generate opposite phase standing waves using optical cavities, to be used as a suppressor of some targeted wavelengths in the transmission spectrum [17]. Depending on the position of cavities relative to the main MIM guiding structure, it is possible to achieve band pass (two MIM waveguides coupled to each other by a circular ring resonator) or band stop filters (laterally coupling ring resonator to an MIM waveguide) [18]. One of the most interesting applications of MIM waveguides that have been the subject of many recent papers is the construction of integrated plasmonic optical filters by cavity waveguide coupling $[19,20]$. Coupled Mode Theory [21] and numerical methods [22] are used for the analysis of such structures. Optical filters, as one of the building blocks of integrated optical circuits, are recently introduced in different geometries such as bidirectional wave coupler based on Bragg Gratings [21,22], bidirectional splitter in different angles of incident light [23], plasmonic splitter based on the MIM waveguide with periodic grooves [24] and wavelength de-multiplexer based on MIM plasmonic nano-capillary resonators [25]. 
The introduced structures suffer from some limitations such as huge dimension for splitting functionality $[21,22$, 24], insulation lack between output ports [23], low transmittance [21-24], power leakage [21-23], and maximum fanout of two for splitter channels [21-24]. An annular ring being able to support multi-modes, should gratify the boundary conditions. Coupling and perturbation methods are used for the resonant mode excitation [23].

In this paper, a novel nanoscale plasmonic filter is introduced while its functionality is verified by numerical simulations in different configurations. Next section describes the dispersion relation for the introduced filter. While the theoretical model is presented in Section 3, Section 4 describes the transmission spectrum results.

\section{Dispersion Relation and Effective Refractive Index of MIM Waveguides}

Figure 1 shows a MIM waveguide, where each metal/ dielectric interface allows SPPs with a TM mode to propagate along $x$ direction. It is shown that whenever the separating space between two interfaces is comparable to the decay lengths of SPPs in the dielectric region, the SPPs get coupled to each other and confined in the dielectric region between two metals [1]. The field analysis inside the dielectric space between two metals could be achieved using Maxwell's equations:

$$
\begin{gathered}
H_{y}^{\text {dielec }}=C_{1} \mathrm{e}^{i \beta x} \mathrm{e}^{k_{1} z}+C_{2} \mathrm{e}^{i \beta x} \mathrm{e}^{-k_{1} z} \\
E_{z}^{\text {dielec }}=\frac{\beta C_{1}}{\omega \varepsilon_{0} \varepsilon_{1}} \mathrm{e}^{i \beta x} \mathrm{e}^{k_{1} z}+\frac{\beta C_{2}}{\omega \varepsilon_{0} \varepsilon_{1}} \mathrm{e}^{i \beta x} \mathrm{e}^{-k_{1} z} \\
E_{x}^{\text {dielec }}=\frac{-i k_{1} C_{1}}{\omega \varepsilon_{0} \varepsilon_{1}} \mathrm{e}^{i \beta x} \mathrm{e}^{k_{1} z}+\frac{i k_{1} C_{2}}{\omega \varepsilon_{0} \varepsilon_{1}} \mathrm{e}^{i \beta x} \mathrm{e}^{-k_{1} z}
\end{gathered}
$$

in which $\beta$ is the propagation constant of the MIM waveguide and $k_{1}=\sqrt{\beta^{2}-k_{0}^{2}}$ is the wave vector normal to the propagation direction and $k_{0}$ is the propagation constant of free space. Using boundary conditions and the field components in the metal region, the dispersion relation of MIM waveguide structure could be calculated by:

$$
\tanh \left(\frac{h}{2} \sqrt{\beta^{2}-k_{0}^{2}}\right)=-\left(\frac{k_{2} \varepsilon_{1}}{\varepsilon_{2} \sqrt{\beta^{2}-k_{0}^{2}}}\right)
$$

where indices 1 and 2 denote the dielectric and metal layers, respectively. Silver is regarded as the metal in this paper, and Drude-Lorentz model with five poles is used for dielectric constant determination [25]:

$$
\varepsilon_{1}(\omega)=1-\frac{\omega_{p}^{2}}{\omega(\omega+i \gamma)}+\sum_{n=1}^{5} \frac{f_{n} \omega_{n}^{2}}{\omega_{n}^{2}-\omega^{2}-i \omega \gamma_{n}}
$$

where $\omega_{p}=2002.6 \mathrm{THz}$ is the plasma frequency and $\gamma=$

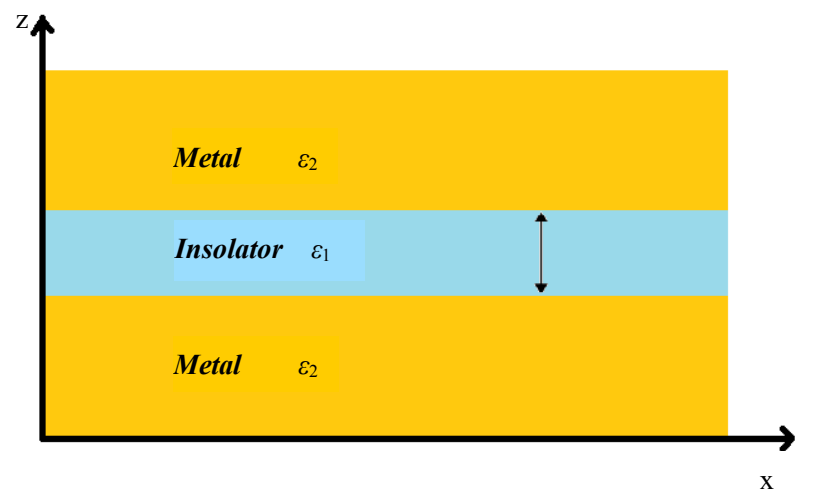

Figure 1. Schematic representation of an MIM structure with two semi-infinite metal slabs of permittivity $\varepsilon_{2}$ surrounding a dielectric layer of thickness $h$ and permittivity $\varepsilon_{1}$.

$11.6012 \mathrm{THz}$ is the damping constant. Table 1 summarizes the main values for dielectric constant calculation, i.e. $\omega_{n}$ resonant frequencies of silver, damping constants $\gamma_{n}$, and oscillator strength $f_{n}[25]$.

\section{Theoretical Model}

The Local Split Resonant (LSR) mode of a circular ring resonator and its effects on the transmission spectra is investigated in this paper theoretically and numerically.

Figure 2 presents the LSR mode excitation using different impedances on the ring resonator. The coupling separation distance $\Delta$ is $10 \mathrm{~nm}$ and the device length $l$ is set to $1 \mu \mathrm{m}$.

The input and output MIM waveguides are coupled by means of a LSR ring resonator made out of two sectors with different impedances. Sector (I) with inside and outside radius of $a_{0}$ and $b_{0}$, and $r_{0}$ as the average $r_{0}=\left(a_{0}+b_{0}\right) / 2$, has a higher characteristic impedance in compare with sector (II) with inside, outside and average radius as $a_{i}, b_{i}$ and $r_{i}=\left(a_{i}+b_{i}\right) / 2$, respectively. Solving the wave equation in cylindrical coordinates, field distribution in region (I) can be written as:

$$
\begin{aligned}
& E_{z}^{(I)}=E_{0}[ J_{\xi}\left(k_{\xi, n}^{I} a_{o}\right) Y_{\xi}\left(k_{\xi, n}^{I} \rho\right) \\
&\left.-J_{\xi}\left(k_{\xi, n}^{I} a_{o}\right) Y_{\xi}\left(k_{\xi, n}^{I} \rho\right)\right] \sin (\xi \varphi) \\
& H_{\rho}^{(I)}=\frac{j \omega \varepsilon_{r}^{(I)} \varepsilon_{0}}{\rho} \frac{\partial E_{z}^{(I)}}{\partial \varphi} \\
& H_{\varphi}^{(I)}=-j \omega \varepsilon_{r}^{(I)} \varepsilon_{0} \frac{\partial E_{z}^{(I)}}{\partial \rho}
\end{aligned}
$$

where, $\xi=p \pi / \varphi_{0}$ for $-\pi / 2<\varphi_{0}<\pi / 2$, and $n$ is the $n$th roots of Bessel functions of the $\xi$ th order which has infinite discrete roots, and $\varepsilon_{r}^{(I)}=\varepsilon_{a i r}$ and $p=1,2,3, \cdots$. In these equations $k_{\xi, n}^{I}$ and $k_{v, n}^{I I}$ are the wave numbers in region (I) and region (II), respectively. Furthermore, 
Table 1. Silver values for evaluating Drude-Lorentz model.

\begin{tabular}{cccc}
\hline $\boldsymbol{f}_{\boldsymbol{n}}$ & $\boldsymbol{\gamma}_{\boldsymbol{n}}(\mathbf{T H z})$ & $\boldsymbol{\omega}_{\boldsymbol{n}}(\mathbf{T H z})$ & $\boldsymbol{n}$ \\
\hline 7.9247 & 939.62 & 197.3 & 1 \\
0.5013 & 109.22 & 1083.5 & 2 \\
0.0133 & 15.71 & 1979.1 & 3 \\
0.8266 & 221.49 & 4392.5 & 4 \\
1.1133 & 584.91 & 9812.1 & 5 \\
\hline
\end{tabular}

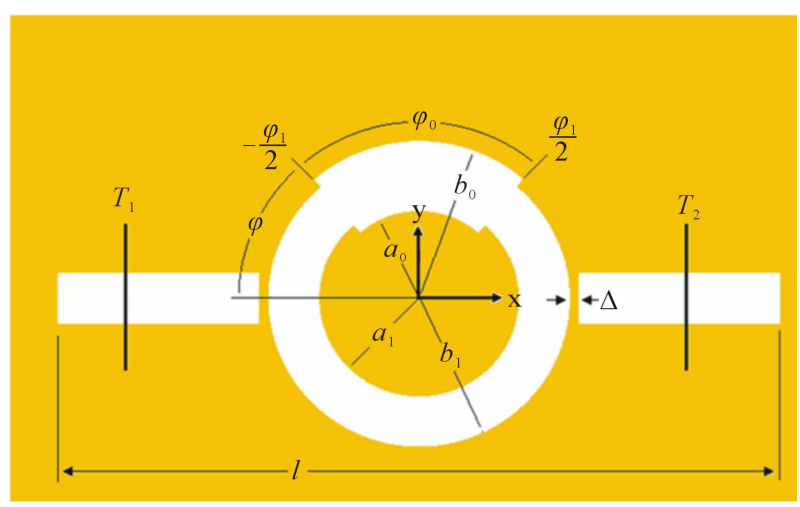

(a)

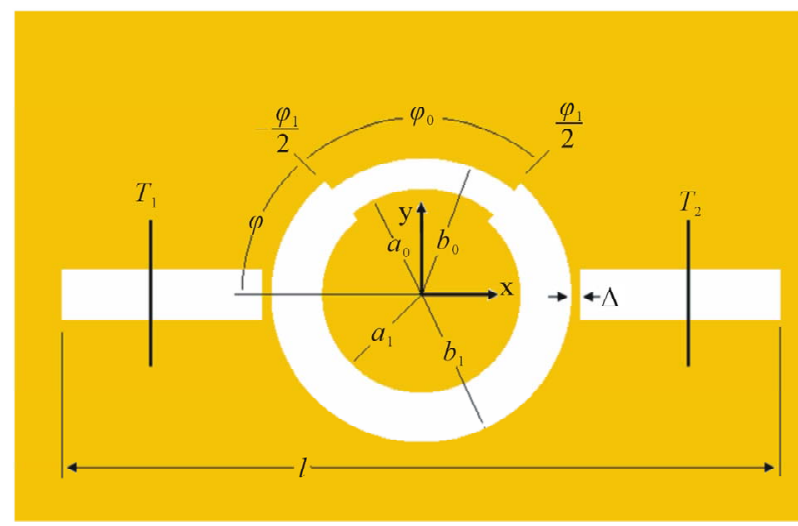

(b)

Figure 2. (a) Schematic of the split mode circular ring resonator with an increasing sector between $-\varphi_{0} / 2$ and $\varphi_{0} / 2$, (b) Schematic of the split mode circular ring resonator with a decreasing sector between $-\varphi_{0} / 2$ and $\varphi_{0} / 2$.

field distribution for region (II) can be calculated from:

$$
\begin{gathered}
E_{z}^{(I I)}=E_{0}\left[J_{v}\left(k_{v, m}^{I I} a_{i}\right) Y_{v}\left(k_{v, m}^{I I} \rho\right)\right. \\
\left.-J_{v}\left(k_{v, m}^{I I} a_{i}\right) Y_{v}\left(k_{v, m}^{I I} \rho\right)\right] \sin (v \varphi) \\
H_{\rho}^{(I I)}=\frac{j \omega \varepsilon_{r}^{(I I)} \varepsilon_{0}}{\rho} \frac{\partial E_{z}^{(I I)}}{\partial \varphi} \\
H_{\varphi}^{(I I)}=-j \omega \varepsilon_{r}^{(I I)} \varepsilon_{0} \frac{\partial E_{z}^{(I I)}}{\partial \rho}
\end{gathered}
$$

where, $\quad v=q \pi /\left(\pi-\varphi_{0}\right) \quad$ for $\quad \pi / 2<\varphi_{0}<3 \pi / 2$, $q=1,2,3, \cdots$, and $m$ is the $m$ th roots of Bessel functions of the $v$ th order. $J_{v}$ and $Y_{v}$ represent the first kind and second kind Bessel function of order $v$, respectively. Using boundary condition at $\rho=a_{i}$ and $\rho=b_{i}$ in region (II), and either one of $\rho=a_{0}$ or $\rho=b_{0}$ for region (I), one obtains:

$$
\begin{aligned}
& \left.\frac{j \omega \varepsilon_{m}^{(I I I)}(\omega) \varepsilon_{0}}{\rho} \frac{\partial E_{z}}{\partial \varphi}\right|_{\rho=a_{i}}=\left.\frac{j \omega \varepsilon_{r}^{(I I)} \varepsilon_{0}}{\rho} \frac{\partial E_{z}}{\partial \varphi}\right|_{\rho=a_{i}} \\
& \left.\frac{j \omega \varepsilon_{m}^{(I I I)}(\omega) \varepsilon_{0}}{\rho} \frac{\partial E_{z}}{\partial \varphi}\right|_{\rho=b_{i}}=\left.\frac{j \omega \varepsilon_{r}^{(I I)} \varepsilon_{0}}{\rho} \frac{\partial E_{z}}{\partial \varphi}\right|_{\rho=b_{i}}
\end{aligned}
$$

and

$$
\begin{aligned}
& \left.\frac{j \omega \varepsilon_{m}^{(I I I)}(\omega) \varepsilon_{0}}{\rho} \frac{\partial E_{z}}{\partial \varphi}\right|_{\rho=a_{o}}=\left.\frac{j \omega \varepsilon_{r}^{(I I)} \varepsilon_{0}}{\rho} \frac{\partial E_{z}}{\partial \varphi}\right|_{\rho=a_{o}} \\
& \left.\frac{j \omega \varepsilon_{m}^{(I I)}(\omega) \varepsilon_{0}}{\rho} \frac{\partial E_{z}}{\partial \varphi}\right|_{\rho=b_{o}}=\left.\frac{j \omega \varepsilon_{r}^{(I I)} \varepsilon_{0}}{\rho} \frac{\partial E_{z}}{\partial \varphi}\right|_{\rho=b_{o}}
\end{aligned}
$$

In addition, using the continuity of tangential components of the magnetic fields at $\varphi=\varphi_{0} / 2$ and $\varphi=-\varphi_{0} / 2$, it can be found that

$$
\begin{gathered}
H_{\varphi}^{(I)}\left(\rho=r_{o}, \varphi=\frac{\varphi_{0}}{2}\right)=H_{\varphi}^{(I I)}\left(\rho=r_{i}, \varphi=\frac{\varphi_{0}}{2}\right) \\
H_{\varphi}^{(I)}\left(\rho=r_{o}, \varphi=-\frac{\varphi_{0}}{2}\right)=H_{\varphi}^{(I I)}\left(\rho=r_{i}, \varphi=-\frac{\varphi_{0}}{2}\right)
\end{gathered}
$$

In conclusion, the characteristic equation is obtained being useful for mode calculation in sector (I).

$$
\frac{J_{v}\left(k_{v, m}^{(I)} b_{o}\right)}{J_{v}\left(k_{v, m}^{(I)} a_{o}\right)}=\frac{Y_{v}\left(k_{v, m}^{(I)} b_{o}\right)}{Y_{v}\left(k_{v, m}^{(I)} a_{o}\right)}
$$

Similarly, characteristic equation for sector (II) can be found as:

$$
\frac{J_{\xi}\left(k_{\xi, n}^{(I I)} b_{i}\right)}{J_{\xi}\left(k_{\xi, n}^{(I I)} a_{i}\right)}=\frac{Y_{\xi}\left(k_{\xi, n}^{(I I)} b_{i}\right)}{Y_{\xi}\left(k_{\xi, n}^{(I I)} a_{i}\right)}
$$

It should be noted that $n=0$ and $m=0$ are not acceptable for all of these equations because of existence of second order Bessel functions in the equations. In fact, each of these sectors follows its individual characteristic equation to illustrate encompass modes in any wavelength. However, instead of using $T M_{v, m}$ and $T M_{\xi, n}$ for each sector, it is preferred to use one notation to specify modes of the whole ring structure which is made of two sectors with different radial widths. Hence, in this study $T M_{v, m}^{\xi, n}$ is defined to show the split mode ring resonator in which, the upper index refers to the mode of the manipulated sector, with angular width of $\varphi_{0}$, and the lower index refers to the mode of the main shape, with angular width of $\pi-\varphi_{0}$ for $\pi / 2<\varphi_{0}<3 \pi / 2$. Using a unique notation is applicable when both $T M_{v, m}$ and $T M_{\xi, n}$ exist. If 
one of these modes does not exist, we cannot use this notation and it is essential to use another one. For example, in this study, if there is no propagating mode inside region (II) or region (I), $T M_{\xi, n}^{(I)}$ and $T M_{v, m}^{(I I)}$, are only used respectively. However, it should be noted that, these resonant modes build a local resonance inside the manipulated sectors and other resonant modes which cannot satisfy the local resonant condition, are lost due to scattering inside the manipulated regions. For example, considering $\varphi_{0}=\pi / 6$, it is anticipated that only the $\xi=6 p$ (integer multiples of sixth) mode maintains the resonant condition and other modes split.

\section{Plasmonic Filter with a LSR Circular Ring Resonator}

\subsection{Typical Band-Pass Filter with a Circular Ring Resonator}

Figure 3 shows a typical plasmonic band-pass filter by the means of a circular ring resonator consisted of two MIM waveguides coupled together by a circular ring resonator [14]. The main parameters are $\Delta, a, b, r$ and $h$ describing the coupling separation and the ring resonator, inside, outside and the average radius of the ring resonator, and the MIM waveguides width, respectively. In this investigation, in order to have fundamental mode $\left(T M_{0}\right)$, $h$ is considered to be $50 \mathrm{~nm}$; to achieve a definitive subwavelength structure. In such structures it is essential to monitor the input signal $\left(T_{1}\right)$, the output signal $\left(T_{2}\right)$, analyzing the power in the frequency domain to reach transmission coefficient, defined as output power divided into input power, $T=P_{\text {out }} / P_{i n}$. Figure 3 shows the transmission spectrum of a typical circular ring resonator with $\Delta$ $=10 \mathrm{~nm}, a=100 \mathrm{~nm}, b=150 \mathrm{~nm}$ as the main property of the filter.

The filter is placed in a length of $L=1 \mu \mathrm{m}$. It can be seen from Figure 3(b) that there exist two resonant peaks, related to two different resonant modes with the wavelengths of $\lambda_{1}=1162 \mathrm{~nm}$ for the first mode, and $\lambda_{2}=602$ $\mathrm{nm}$ for the second mode. Considering $r_{i}$ and $r_{o}$ as the inside and outside radius of the ring resonator, respectively, it is shown that these modes can be computed using the following equation [23]:

$$
\frac{J_{n}^{\prime}\left(k r_{o}\right)}{Y_{n}^{\prime}\left(k r_{o}\right)}-\frac{J_{n}^{\prime}\left(k r_{i}\right)}{Y_{n}^{\prime}\left(k r_{i}\right)}=0
$$

in which $k=\omega / c\left(\sqrt{\mu_{r} \varepsilon_{r}}\right)$ is the wave number, $c=1 /\left(\sqrt{\mu_{0} \varepsilon_{0}}\right), \varepsilon_{r}$ and $\mu_{r}$ are light speed in free space, relative permittivity and permeability, respectively. It can be shown that the effective refraction index of a MIM structure can be computed as [1]:

$$
n_{\text {eff }}=\frac{\beta}{k_{0}}=\frac{\operatorname{Re}[\beta]+i \operatorname{Im}[\beta]}{k_{0}}=\operatorname{Re}\left[n_{\text {eff }}\right]+i \operatorname{Im}\left[n_{\text {eff }}\right]
$$

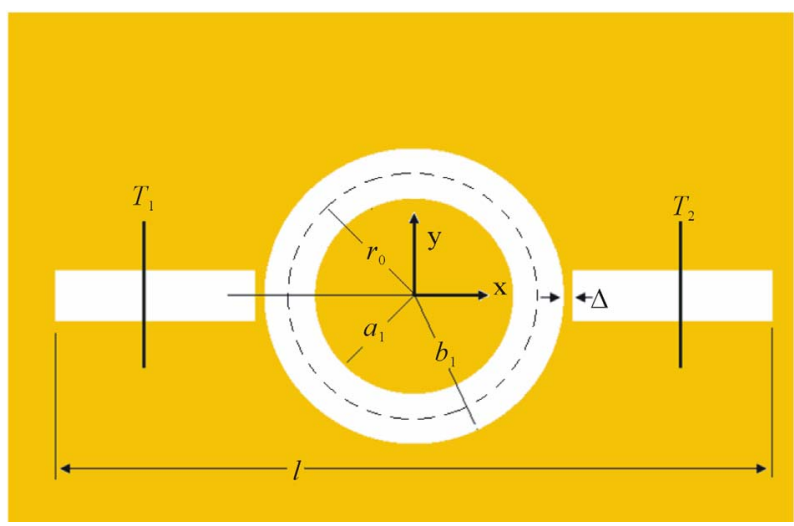

(a)

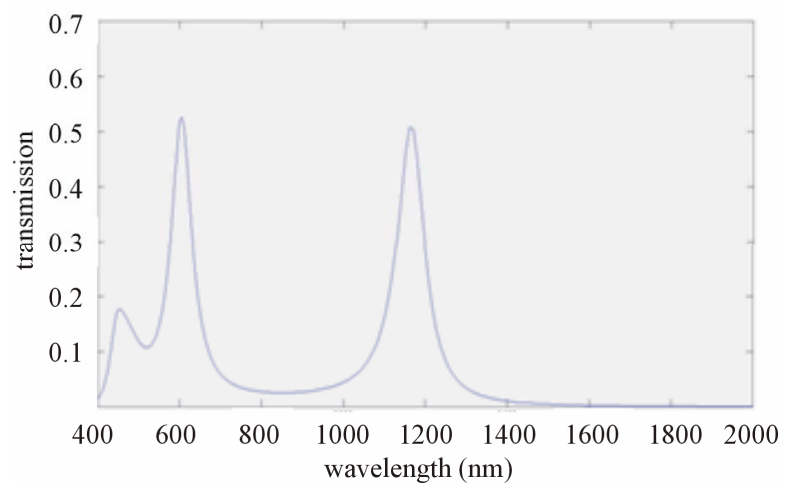

(b)

Figure 3. (a) Schematic of a typical plasmonic MIM bandpass filter using a circular ring resonator, (b) The transmission spectrum of a typical plasmonic MIM band-pass filter using a circular ring resonator.

The transmission simulation of the filter is calculated using 2D FDTD and is shown in Figure 3(b). In these simulations, it is assumed that the size of each uniform Yee cell of the structure in $x$ and $z$-directions are $\Delta x=\Delta z$ $=2 \mathrm{~nm}$. The computation domain of this structure is a conventional perfectly matched layer (CPML) absorbing boundary condition.

\subsection{Adjustable Plasmonic Filter Using a Split Mode Ring Resonator}

This section investigates the effect of using a split mode ring resonator on the main modal characteristics. In fact, the split mode resonator is made out of two or more changed impedance circular sectors on the circular ring. Due to impedance changing, a local resonant boundary condition is created which can reserve or split the different mode's energy. It is assumed for simplicity that the constant coupling distance $\Delta$, was setting as that in the investigated structure (see Figure 2).

In order to study the effect of the position of the manipulated sector on the transmission spectrum of the ring resonator filter, one can observe Figure 4 in which $\varphi_{0}$ is 


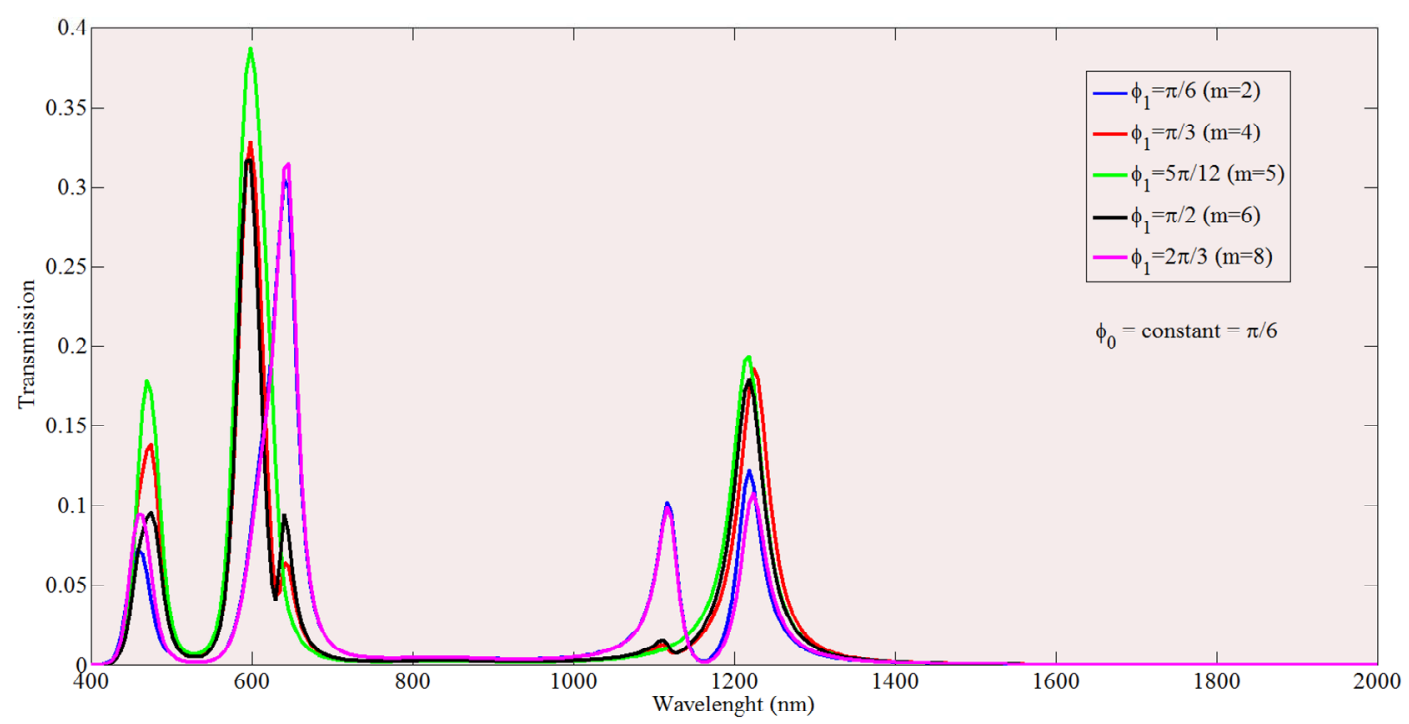

(a)

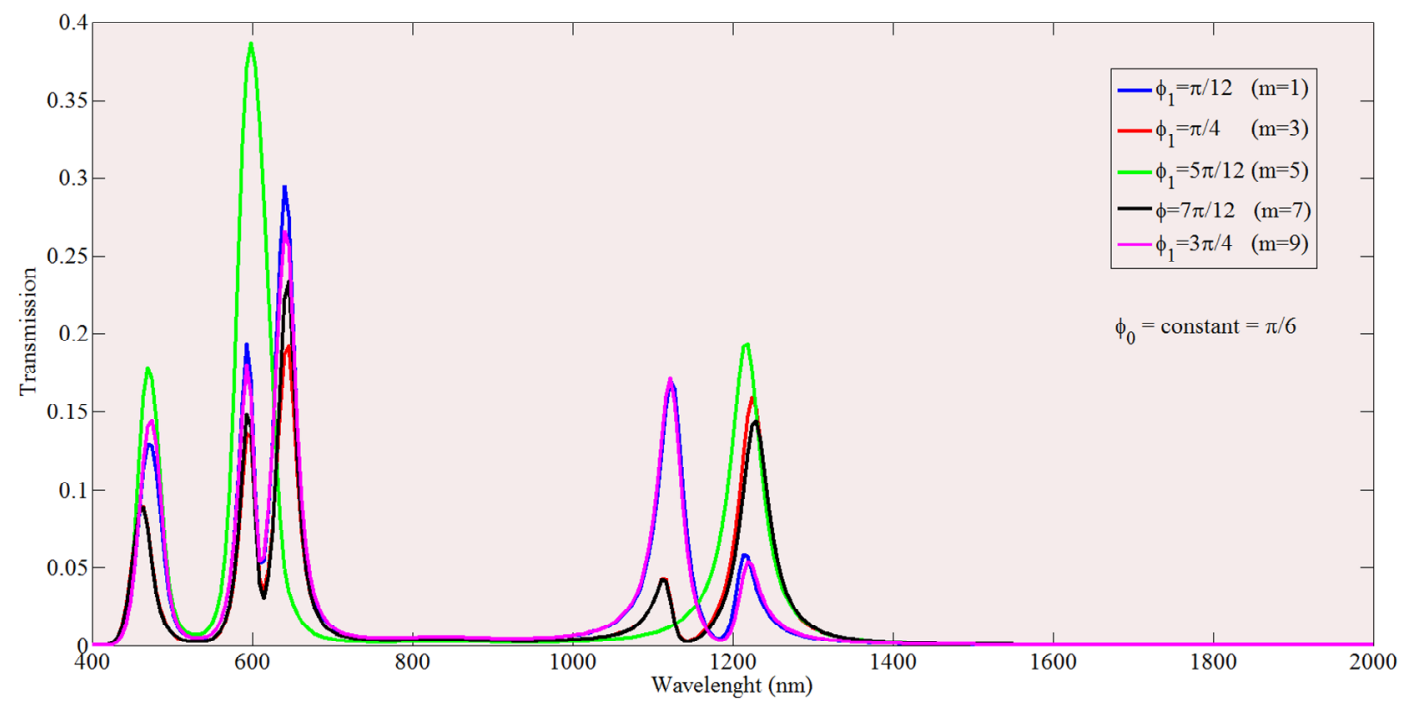

(b)

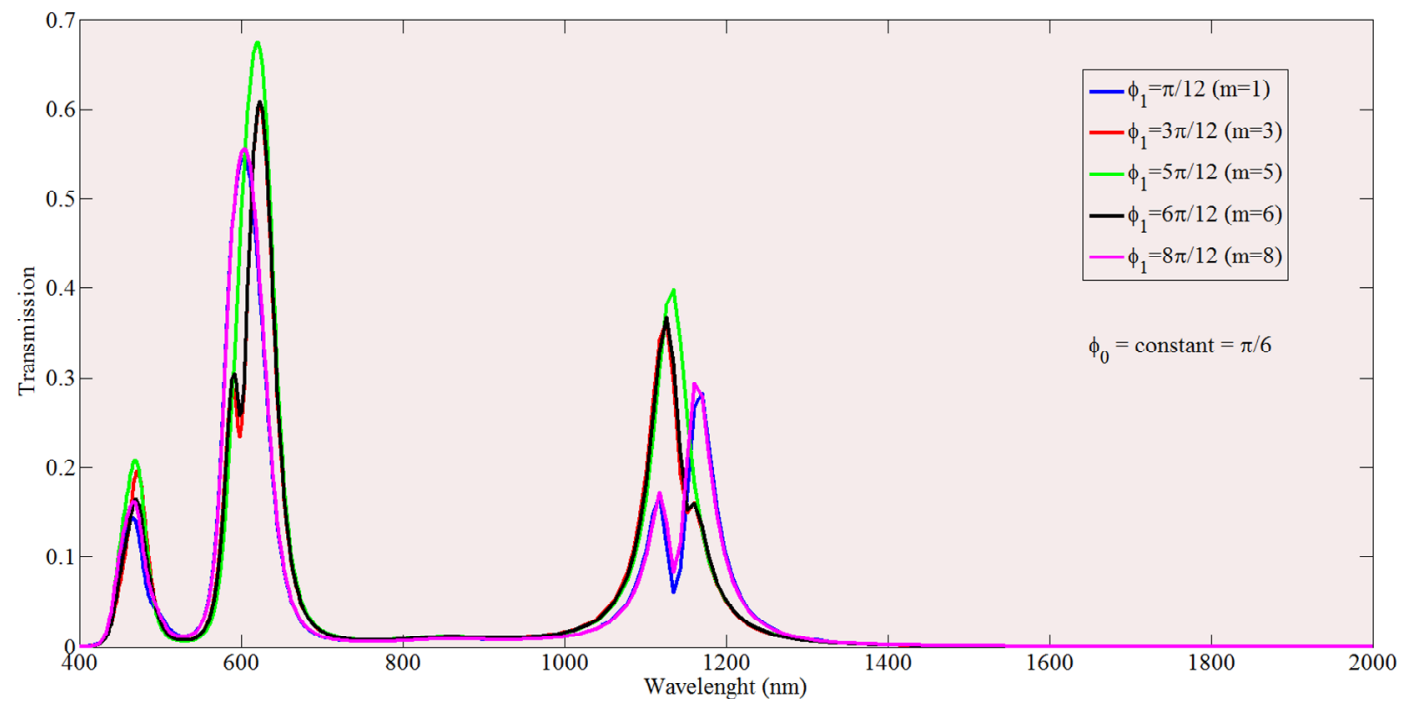

(c) 


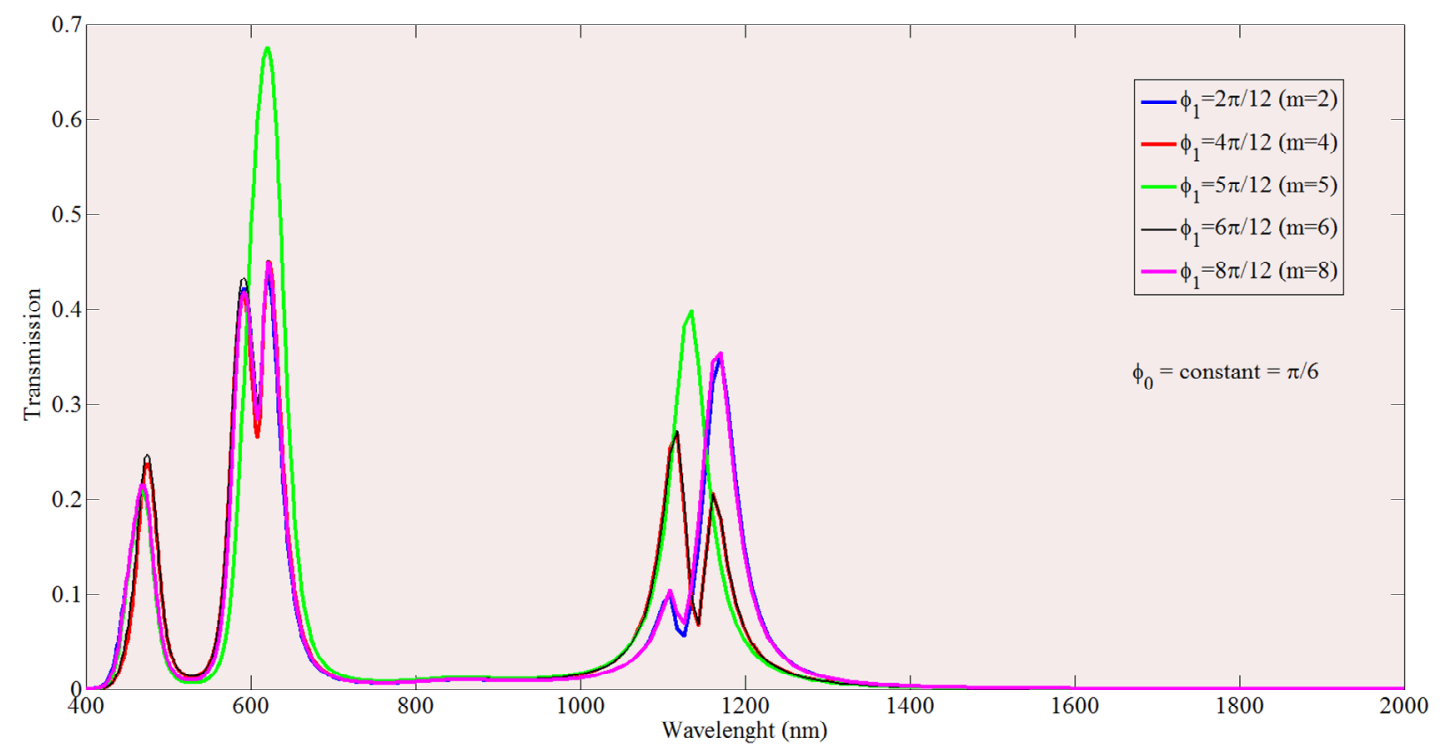

(d)

Figure 4. (a) The transmission spectrum of the split mode ring resonator plasmonic filter for even values of $\mathbf{m}$ in an decreasing split sector with $a_{0}=110 \mathrm{~nm}, b_{0}=140 \mathrm{~nm}$, constant $\varphi_{0}=\pi / 3$ and alternating $\varphi_{1}$, (b) The transmission spectrum of the split mode ring resonator plasmonic filter for odd values of $\mathrm{m}$ in an decreasing split sector with $a_{0}=110 \mathrm{~nm}, b_{0}=140 \mathrm{~nm}$, constant $\varphi_{0}=\pi / 3$ and alternating $\varphi_{1}$, (c) The transmission spectrum of the split mode ring resonator plasmonic filter for some odd values of $\mathrm{m}$ in an increasing split sector with $a_{0}=90 \mathrm{~nm}, b_{0}=160 \mathrm{~nm}$, constant $\varphi_{0}=\pi / 3$ and alternating $\varphi_{1}$, (d) The transmission spectrum of the split mode ring resonator plasmonic filter for even values of $\mathrm{m}$ in an increasing split sector with $a_{0}=90 \mathrm{~nm}$, $b_{0}=160 \mathrm{~nm}$,constant $\varphi_{0}=\pi / 3$ and alternating $\varphi_{1}$.

constant and $\varphi_{1}$ is varying relative to the $x$-axis, as the port's main axis. In this study in order to investigate the manipulated position dependency of the sector, it is assumed that $\varphi_{1}=m \pi / 12, \quad m=1,2,3, \cdots$. Figure 4(c) shows that in the expanded sector case (for example $a_{0}=$ $90 \mathrm{~nm}$ and $b_{0}=160 \mathrm{~nm}$ ) and for odd $m$ numbers, the transmission spectrum amplitude for all modes are increased by approaching $\varphi_{1}$ to $5 \pi / 12$. In addition, this figure shows that for the sector which is placed nearby the ports, the first mode disappears and two new resonant modes are created in return and a valley appears. The valleys become deeper by decreasing the distance of sector expansion to the ports.

The effect of even $m$ numbers is shown in Figure 4(d). It is obvious that by approaching the manipulated sector to the ports, each of the original first and second resonant modes split into two new lateral resonant modes. In addition, the transmission spectrum for $m=5$ is shown in Figures 4(c) and (d) to demonstrate the transmission spectrum when $\varphi_{1}$ gets close to $5 \pi / 12$ for which, the manipulated sector is homolographic around $y$ axis. In other words, the influence of increasing $m$ on the transmission spectrum is a periodic process. Hence, it is essential to analyze the transmission spectrum using even/odd $m$.

As the center of the sector approaches $\pi / 2$, the transmission amplitude of the first mode of the splitted modes increases and the amplitude of the second mode decreases gradually. Finally, for $m=5$, for which the sector is symmetric around $y$ axis, the transmission amplitude takes a resonant peak which is the mean value of the neighboring amplitudes. Figure 4(b) illustrates the effect of odd $m$ values on the transmission spectrum. It is obvious that, splitting is occurred and a large increase of the transmission amplitude is observed for the second resonant mode and for all $m$ values. However, splitting phenomena affects the second resonant mode less severe in comparison with the first mode. In addition, according to Figure 4(b), it is found that, unlike previous discussion for even values of $m$, the augmenting of $\varphi_{1}$ will not end in the enhancement of second mode transmission amplitude. Alternating the width of the manipulated sector could play an important role on transmission spectrum. Figure 5 depicts the transmission spectrum, affected by changing $\varphi_{0}$ of manipulated sector, respectively. In order to study the effects of changing $\varphi_{0}$ on transmission spectrum, first we consider the narrowing sector case with $a_{0}$ $=110 \mathrm{~nm}, b_{0}=140 \mathrm{~nm}$ and $\varphi_{1}=0$ and then we investigate the effect of changing $\varphi_{0}$. Moreover, this effect is investigated for the case that the sector is symmetric around $y$ axis. According to Figure 5(a), the second resonant mode of a typical ring resonator is splitted into two different resonant peaks. By enhancing $\varphi_{0}$ from $2 \pi / 12$ to $\pi / 2$, the amplitude of the second peak becomes equal to the first peak and finally, as $\varphi_{0}$ gets closer to $\pi / 2$, the first peak vanishes gradually and only the second peak with a great amplitude remains. In addition, $\varphi_{0}$ enhancement 


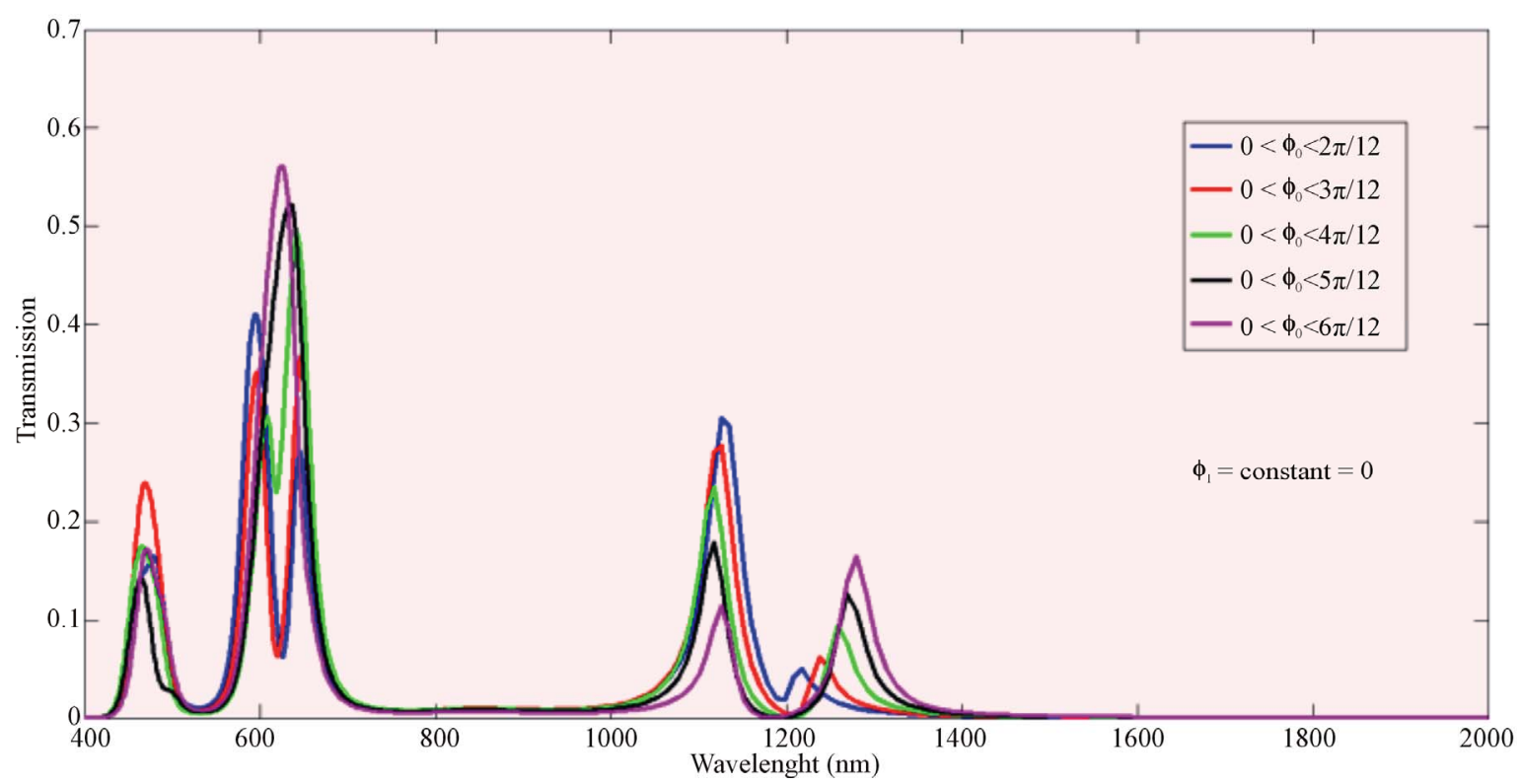

(a)

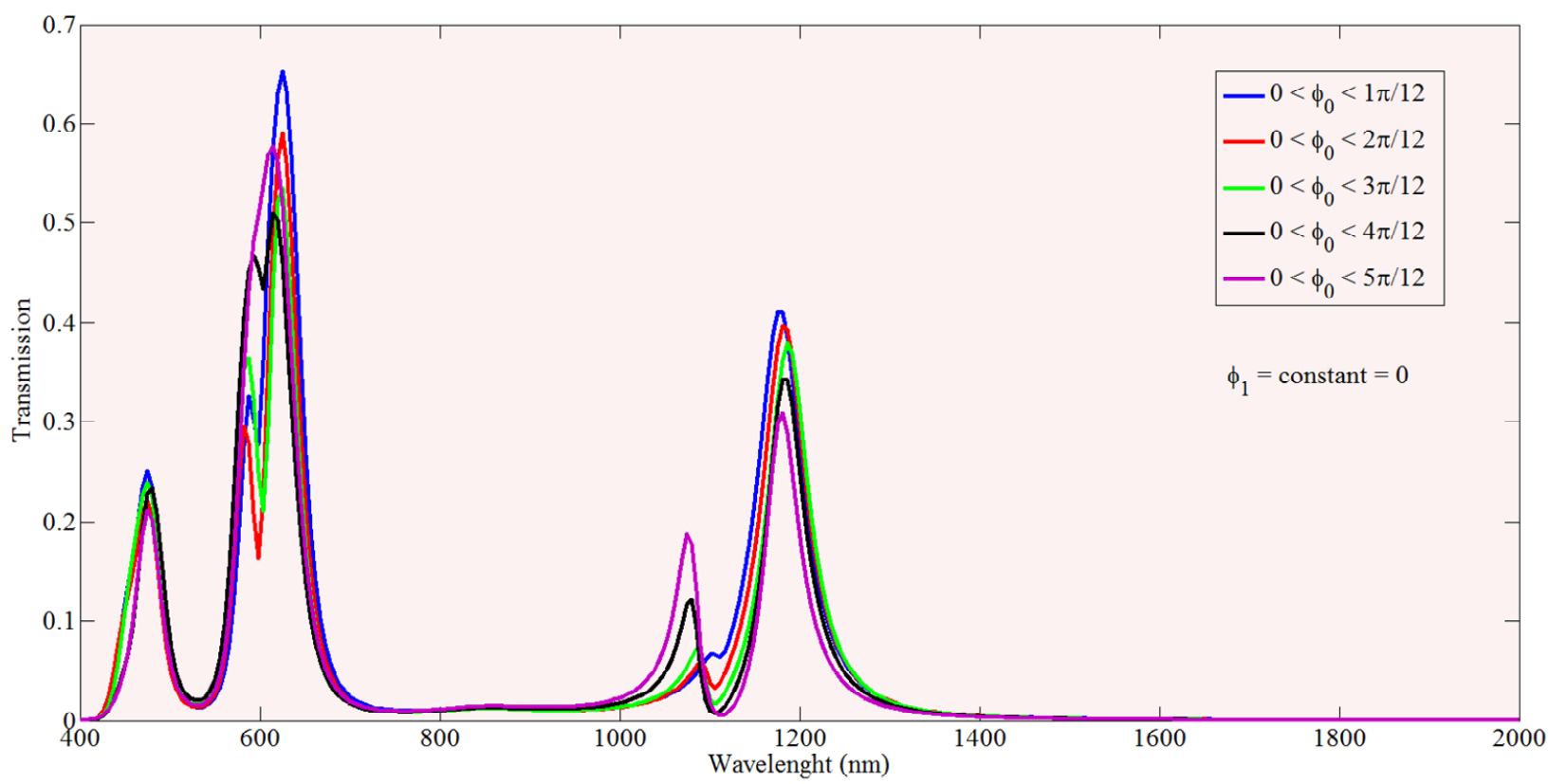

(b)

Figure 5. (a) The transmission spectrum of the split mode ring resonator plasmonic filter with $\varphi_{1}$ constant and changing $\varphi_{0}$ for decreasing sector, (b) The transmission spectrum of the split mode ring resonator plasmonic filter with $\varphi_{1}$ constant and changing $\varphi_{0}$ for increasing sector.

results in reducing the amplitude of the first resonance and generating a new quasi-first order resonant mode with a less amplitude in compare with the previous one.

Another parameter which affects the operation of a split ring resonator is the changing of the radial width of the manipulated sector. Figure $\mathbf{6}$ depict the transmission spectrum in the case that the radial width of the manipulated sector takes different values.

It is concluded from Figure 6 that augmenting the ra- dial width of the enhanced sector will end in a red shift for the second resonance, which is a significant property of integrated nano-optic circuits. In other words, using a sector with a small expanded radial width with angular width of $\pi / 6$ and $\varphi_{1}=5 \pi / 12$, it is possible to reach higher resonant wavelengths (or more size reduced circuits) without the whole structure scaling.

Figure 7, shows the real $\left(H_{y}\right)$ field profile of the decreased split mode ring resonators for three different 


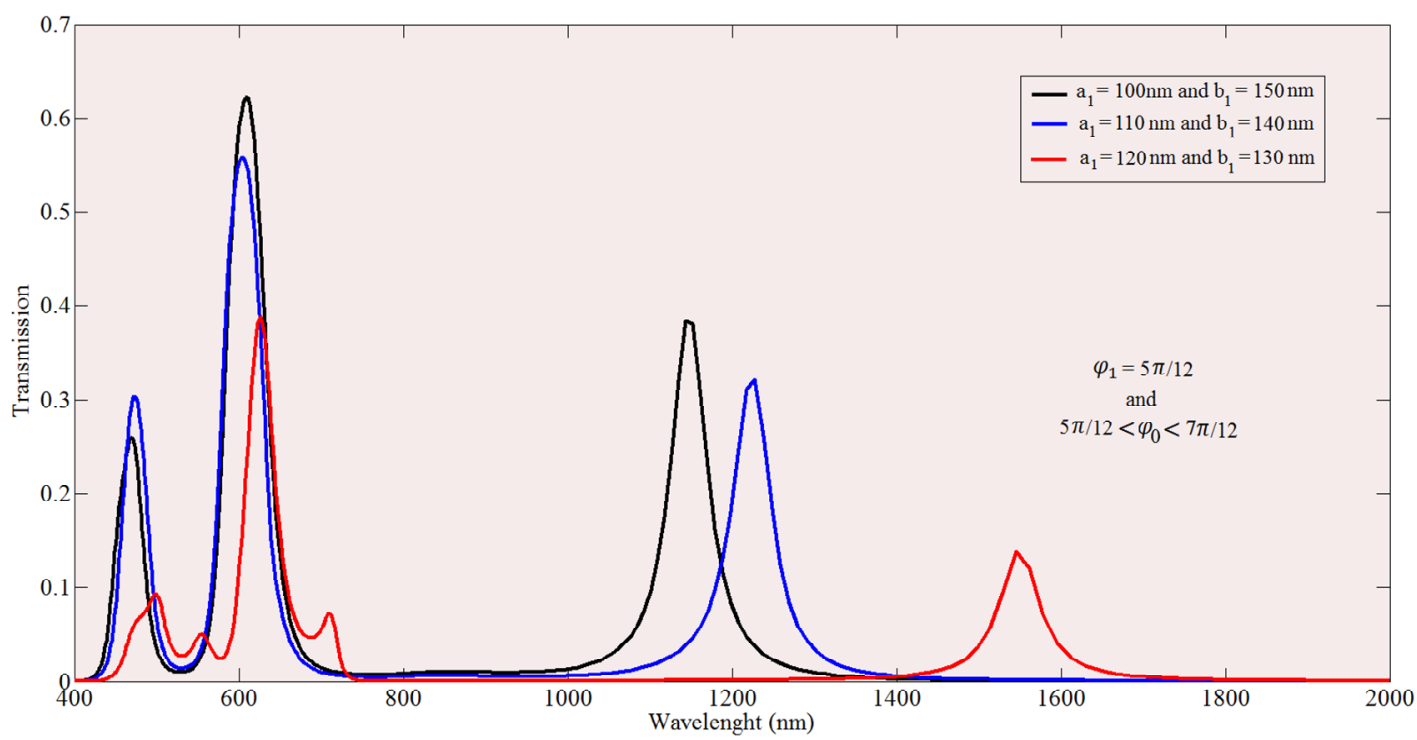

Figure 6. The transmission spectrum of a split mode ring resonator for an increased sector with $\varphi_{1}=5 \pi / 12$ and $5 \pi / 12<\varphi_{0}<$ $7 \pi / 12$, and varying $a_{0}$ and $b_{0}$.
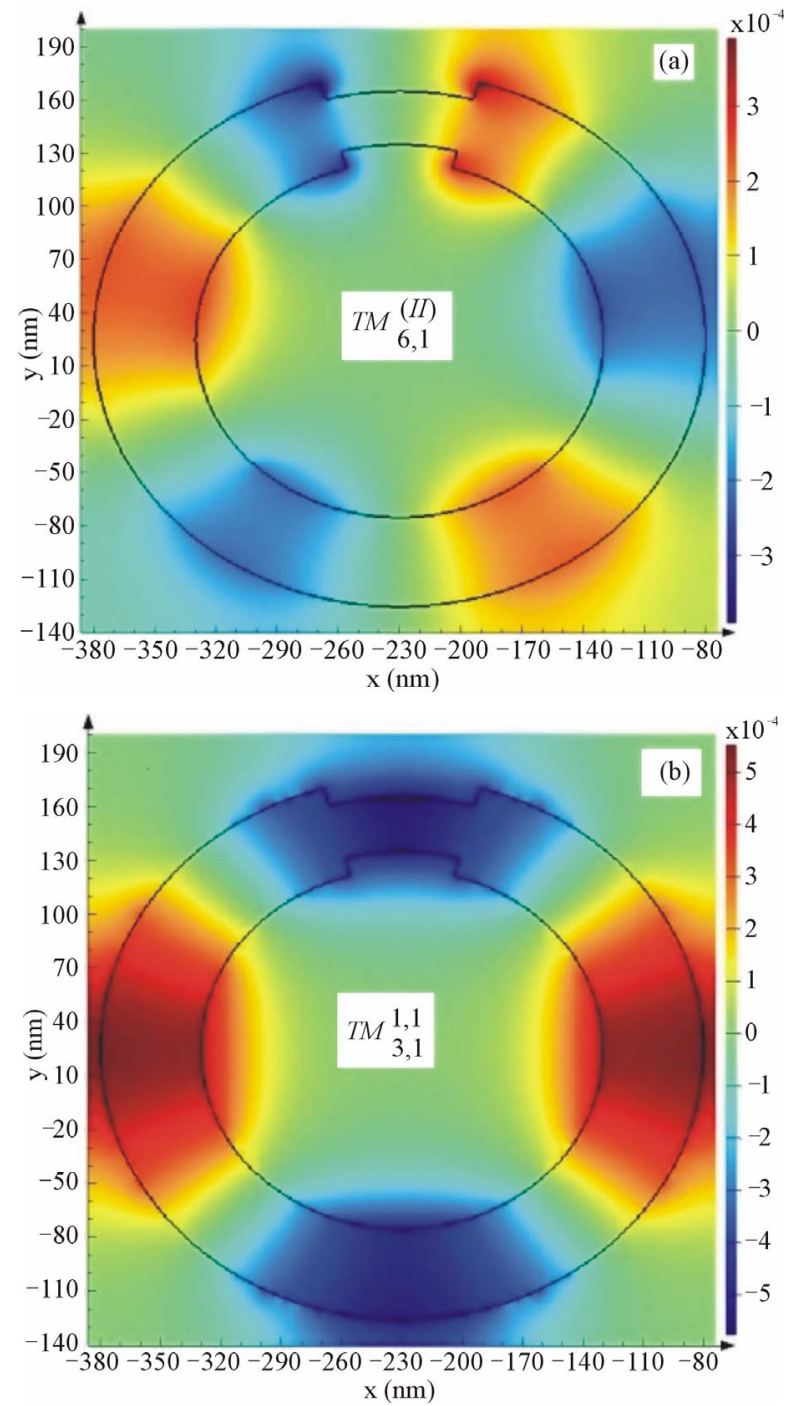

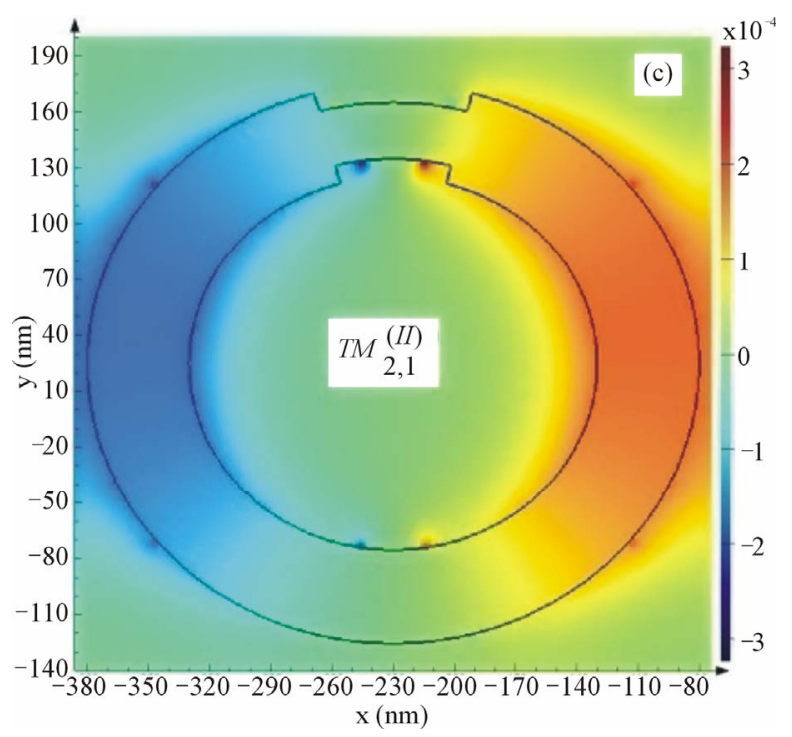

Figure 7. (a) The Real (Hy) field profile of the decreased split mode ring resonator of $\varphi_{0}=\pi / 6, \varphi_{1}=5 \pi / 12, a_{0}=110$ $\mathrm{nm}$ and $b_{0}=140 \mathrm{~nm}$ for $\lambda=476.5 \mathrm{~nm}$, (b) $\lambda=608.3 \mathrm{~nm}$, (c) $\lambda$ $=1228 \mathrm{~nm}$.

wavelengths.

\section{Conclusion}

A novel nanoscale plasmonic band-pass filter is proposed based on MIM waveguide and circular ring resonator. The filter is formed by replacing a sector of ring resonator with a different size part. Two main structures are simulated by the filter, named as narrowing parts and expanded parts. Splitting the resonant modes, redshift in the modes and maximum transmission modulation is observed by changing the manipulated duty cycle, its 
location, and the manipulation thickness.

\section{Acknowledgements}

The authors would like to thank Iranian Nanotechnology Initiative Councilman and Professor Ali Beitollahi for their support.

\section{REFERENCES}

[1] S. I. Bozhevolnyi, "Plasmonic Nanoguides and Circuits," In: S. I. Bozhevolnyi, Ed., Plasmonics and Metamaterials, Pan Stanford Publishing, Singapore City, 2008. http://dx.doi.org/10.1142/9789814241335

[2] H. Raether, "Surface Plasmon Smooth and Rough Surfaces and on Gratings," Springer-Verlag, Berlin, 1988.

[3] E. Ozbay, "Plasmonics: Merging Photonics and Electronics at Nanoscale Dimensions," Science, Vol. 311, No. 5758, 2006, pp. 189-193.

http://dx.doi.org/10.1126/science.1114849

[4] S. A. Maier, P. G. Kik, H. A. Atwater, S. Meltzer, E. Harel, B. E. Koel and A. G. Requicha, "Local Detection of Electromagnetic Energy Transport below the Diffraction Limit in Metal Nanoparticle Plasmon Waveguides," Nature, Vol. 2, No. 4, 2003, pp. 229-232.

http://dx.doi.org/10.1038/nmat852

[5] M. Quinten, A. Leitner, J. R. Krenn and F. R. Aussenegg, "Electromagnetic Energy Transport via Linear Chains of Silver Nanoparticles," Optics Letters, Vol. 23, No. 17, 1998, pp. 1331-1333. http://dx.doi.org/10.1364/OL.23.001331

[6] S. A. Maier, P. G. Kik and H. A. Atwater, "Optical Pulse Propagation in Metal Nanoparticle Chain Waveguides," Physical Review B, Vol. 67, No. 20, 2003, Article ID: 205402. http://dx.doi.org/10.1103/PhysRevB.67.205402

[7] D. Martin-Cano, M. L. Nesterov, A. I. Fernandez-Dominguez, F. J. Garcia-Vidal, L. Martin-Moreno and Esteban Moreno, "Domino Plasmons for Sub Wavelength Terahertz Circuitry," Optics Express, Vol. 18, No. 1, 2010, pp. 754-764. http://dx.doi.org/10.1364/OE.18.000754

[8] D. F. P. Pile and D. K. Gramotev, "Channel Plasmon-Polariton in a Triangular Groove on a Metal Surface," $O p$ tics Express, Vol. 29, No. 10, 2004, pp. 1069-1071.

http://dx.doi.org/10.1364/OL.29.001069

[9] S. I. Bozhevolnyi, V. S. Volkov, E. Devaux and T. W. Ebbesen, "Channel Plasmon-Polariton Guiding by SubWavelength Metal Grooves," Physical Review Letters, Vol. 95, No. 4, 2005, Article ID: 046802. http://dx.doi.org/10.1103/PhysRevLett.95.046802

[10] D. K. Gramotev and D. F. P. Pile, "Single-Mode SubWavelength Waveguide with Channel Plasmon-Polaritons in Triangular," Applied Physics Letters, Vol. 85, 2004, pp. 6323-6325. http://dx.doi.org/10.1063/1.1839283

[11] E. Verhagen, J. A. Dionne, L. Kuipers, H. A. Atwater and A. Polman, "Near-Field Visualization of Strongly Confined Surface Plasmon-Polaritons in Metal-Insulator-Metal
Waveguides," NanoLetters, Vol. 8, No. 9, 2008, pp. 2925-2929. http://dx.doi.org/10.1021/nl801781g

[12] J. A. Dionne, L. A. Sweatlock, H. A. Atwater and A. Polman, "Plasmon Slot Waveguides: Towards Chip-Scale Propagation with Subwavelength-Scale Localization," Physical Review B, Vol. 73, No. 3, 2006, Article ID: 035407. http://dx.doi.org/10.1103/PhysRevB.73.035407

[13] Y. Matsuzaki, T. Okamoto, M. Haraguchi, M. Fukui and M. Nakagaki, "Characteristics of Gap Plasmon Waveguide with Stub Structures," Optics Express, Vol. 16, No. 21, 2008, pp. 16314-16325.

http://dx.doi.org/10.1364/OE.16.016314

[14] T. B. Wang, X. W. Wen, C. P. Yin and H. Z. Wang, "The Transmission Characteristics of Surface Plasmon Polaritons in Ring Resonator," Optics Express, Vol. 17, No. 26, 2009, pp. 24096-24101. http://dx.doi.org/10.1364/OE.17.024096

[15] X. Lin and X. Huang, "Tooth-Shaped Plasmonic Waveguide Filter with Nanometric Sizes," Optics Express, Vol. 33, No. 23, 2008, pp. 2874-2876. http://dx.doi.org/10.1364/OL.33.002874

[16] J. Tao, X. G. Huang, X. Lin, Q. Zhang and X. Jin, “A Narrow-Band Subwavelength Plasmonic Waveguide Filter with Asymmetrical Multiple Teeth-Shaped Structure," Optics Express, Vol. 17, No. 16, 2009, pp. 13989-13994. http://dx.doi.org/10.1364/OE.17.013989

[17] P. H. Lee and Y. C. Lan, "Plasmonic Waveguide Filters Based on Tunneling and Cavity Effects," Springer, Vol. 5, 2010, pp. 417-422.

[18] A. Setayesh, S. R. Mirnaziry and M. S. Abrishamian, "Nu-Merical Investigation of Tunable Band-Pass $\backslash$ BandStop Plasmonic Filters with Hollow-Core Circular Ring Resonator," Optical Society of Korea, Vol. 15, No. 1, 2011, pp. 82-89. http://dx.doi.org/10.3807/JOSK.2011.15.1.082

[19] A. Hosseini and Y. Massoud, "Nanoscale Surface Plasmon Based Resonator Using Rectangular Geometry," Applied Physical Letters, Vol. 90, No. 18, 2007, Article ID: 181102. http://dx.doi.org/10.1063/1.2734380

[20] A. V. Krasavin and A. V. Zayats, "Electro-Optic Switching Element for Dielectric-Loaded Surface Plasmon Polariton Waveguides," Applied Physical Letters, Vol. 96, No. 4, 2010, Article ID: 041107. http://dx.doi.org/10.1063/1.3464552

[21] Q. Li, T. Wang, Y. Su, M. Yan and M. Qiu, "Coupled Mode Theory Analysis of Mode-Splitting in Coupled Cavity System," Optics Express, Vol. 18, No. 8, 2010, pp. 8367-8382. http://dx.doi.org/10.1364/OE.18.008367

[22] H. Lu, X. Liu, D. Mao, L. Wang and Y. Gong, "Tunable Band-Pass Plasmonic Waveguide Filters with Nanodisk Resonators," Optics Express, Vol. 18, No. 17, 2010, pp. 17922-17927. http://dx.doi.org/10.1364/OE.18.017922

[23] I. Wolff and N. Knoppik, "Microstrip Ring Resonator and Dispersion Measurement on Microstrip Lines," Electronics Letters, Vol. 7, No. 26, 1971, pp. 779-781.

http://dx.doi.org/10.1049/el:19710532

[24] A. Taflove and S. C. Hagness, "Computational Electrodynamics: The Finite-Difference Time-Domain Method," 
Artech House, Norwood, 2005.

[25] A. Pannipitiya, I. D. Rukhlenko, M. Haroldo, T. Hattori and G. P. Agrawal, "Improved Transmission Model for
Metal-Dielectric-Metal Plasmmonic Waveguides with Stub Structures," Optics Express, Vol. 18, No. 6, 2010, pp. 6191-620. http://dx.doi.org/10.1364/OE.18.006191 